\title{
THE CORE OF A REINSURANCE MARKET*
}

\author{
Berratd Baton and Jenn I.emalre \\ Université Libre de Bruxelles
}

\begin{abstract}
In a series of celebrated papers, K. Borch characterized the set of the Paretooptimal risk exchange treaties in a reinsurance market. However, the Paretooptimality and the individual rationality conditions, considered by Borch, do not preclucle the possibility that a coalition of companies might be better off by seceding from the whole group. In this paper, we introduce this collective rationality condition and characterize the core of this game without transferable utilities in the important special case of exponential utilities. The mathematical conditions we obtain can be interpreted in terms of insurance premiums, calculated by means of the zero-utility premium calculation principle. We then show that the core is always non-void and conclude by an example.
\end{abstract}

\section{UTILITY FUNCTIONS IN INSURANCE}

Utility functions were introduced into the actuarial world by BoRCH (1961). This notion was since then used mainly in two specific insurance models:

\section{The principle of zero-utility}

Introduced by BÜHLMANN (1970), this premium calculation principle requires equality of the company's utility before and after signature of an insurance policy. Denoting by $R_{j}$ the free reserves, $P_{j}$ the premium (to be calculated), $F_{j}\left(x_{j}\right)$ the distribution function of the total claim amount $\xi_{j}$, and $u_{j}\left(x_{j}\right)$ the utility of the amount $x_{j}$ obtained with certainty, for a given company $C_{j}$, the principle demands that

$$
u_{j}\left(R_{j}\right)=\int_{0}^{\infty} u_{j}\left(R_{j}+P_{j}-x_{j}\right) d F_{j}\left(x_{j}\right)
$$

Many authors, among which GERBER (1974a, 1974b) and LEEPIN (1975) have shown that the exponential utility functions

$$
u_{f}\left(x_{j}\right)=\frac{1}{c_{j}}\left(1-e^{-c_{j} x_{j}}\right), \quad\left(c_{j} \geqslant 0\right)
$$

characterized by a constant risk aversion

$$
r_{j}(x)^{\cdot}=\frac{-u_{j}^{\prime \prime}(x)}{u_{j}^{\prime}(x)}=c_{j},
$$

* This paper was greatly improved after successive presentations at the Eidgenössische Technische Hochschule in Zürich, the University of California at Berkeley and the Oberwolfach Mecting on Risk Theory. 
possess very desirable properties. In that case the premium can be explicitly computed; one obtains

$$
P_{j}=\frac{1}{c_{j}} \log M_{j}\left(c_{j}\right)
$$

where $M_{j}\left(c_{j}\right)$ is the moment-generating function of $S_{j}$ calculated at point $c_{j}$. $P_{j}$ will be referred to in the sequel as the exponential utility premium.

\section{A Model of risk exchange}

Introduced by Borch (1960a, 1960b, 1962), this model considers a pool of $n$ insurance companies $\left(C_{1}, \ldots, C_{n}\right)$, willing to improve their secureness by means of an exchange of risks treaty. Let $\left[R_{f}, F_{j}\left(x_{j}\right)\right]$ be the initial situation of $C_{j}$, evaluated by its expected utility

$$
U_{j}\left(x_{j}\right)=U_{j}\left[R_{j}, F_{j}\left(x_{j}\right)\right]=\int_{0}^{\infty} u_{j}\left(R_{j}-x_{j}\right) d F_{j}\left(x_{j}\right) .
$$

The mombers of the pool will try to increase their utilities by concluding a treaty

$$
\bar{y}=\left[y_{1}\left(x_{1}, \ldots, x_{n}\right), \ldots, y_{n}\left(x_{1}, \ldots, x_{n}\right)\right],
$$

where $y_{j}^{\prime}\left(x_{1}, \ldots, x_{n}\right)=y_{j}(\bar{x})$ is the sum $C_{j}$ has to pay if the claims for the different companies respectively amount to $x_{1}, \ldots, x_{n}$.

Since all the claims must be indemnified, the treaty has to satisfy the admissibility condition

Condition 1: Admissibility

$$
\sum_{j=1}^{n} y_{j}(\bar{x})=\sum_{j=1}^{n} x_{j}=z
$$

the total amount of the claims. After the signature of $\tilde{y}$, the utility of $C_{j}$ becomes

$$
U_{j}(\bar{y})=\int_{\Theta_{N}} u_{j}\left[R_{j}-y_{j}(\bar{x})\right] d F_{N}(\bar{x}),
$$

where $\theta_{N}$ is the positive orthant of $E^{n}$ and $F_{N}(\bar{x})$ the $n$-dimensional distribution function of the claims $\bar{x}=\left(x_{1}, \ldots, x_{n}\right)$.

\section{Condition 2: P'areto-optimality}

A treaty $\bar{y}$ is efficient or Pareto-optimal if there is no $\bar{y}^{\prime}$ such that $U_{j}\left(\bar{y}^{\prime}\right) \geqslant$ $U_{j}(\bar{y})$ for all $j$, with at least one strict inequality. Du Mouchel (1968) has charactcrized the Pareto-optimal trcaties by means of the following theorem. 


\section{Theorcm 1}

Providing all utility functions are such that $u_{j}^{\prime}(x)>0$ and $u_{j}^{\prime \prime}(x) \leqslant 0$, a treaty $\bar{y}$ is Pareto-optimal if and only if there exists $n$ non-negative constants $k_{1}=1$, $k_{2}, \ldots, k_{n}$, such that, with probability 1 ,

$$
k_{j} u_{j}^{\prime}\left[R_{j}-y_{j}(\bar{x})\right]=k_{1} u_{1}^{\prime}\left[R_{1}-y_{1}(\bar{x})\right] \quad j=1, \ldots, n .
$$

Let $K=\left\{k_{1}, \ldots, k_{n}\right\}$. Using very mild technical conditions, it is not difficult to show [DU Mouchel (1968), Lemaire (1973)] that one and only one Paretooptimal treaty always exists for given $K$. However, there usually exists an infinity of $K$ that satisfy (1) and (2), even when one takes into consideration the fact that no company will enter the pool if its utility is decreased:

\section{Condition 3: Individual rationality}

For all $j=1, \ldots, n \quad U_{j}(\bar{y}) \geqslant U_{j}\left(x_{j}\right)$.

The non-uniqueness of the solution is easily explained by the fact that no sharing rule appears in the definition of Pareto-optimality. Cooperation increases global welfare, and nothing is said about the way the companies will divide the benefits of their mutual agreement. The different admissible values of $K$ correspond to all the possible ways of sharing the profits; each company has interest to obtain a $k_{j}$ as high as possible, in ordcr to pay as less as possible. The interests of the members of the pool are thus partially complementary (as a whole, the group will prefer a Pareto-optimal treaty), and partially conflicting (eacl company will have to bargain over its constant $k_{f}$ ). This is characteristic of a game-theoretic situation; indeed, it has been shown by LEMAIRE (1973) that the risk exchange market is in fact a game without transferable utilities.

In the case of exponential utilities, the solution of (2), with the constraint (1), is a familiar quota-share treaty

$$
y_{j}(\bar{x})=q_{j} z+y_{j}(0), \quad \text { with }\left\{\begin{array}{l}
q_{j}=\frac{1 / c_{j}}{\sum_{i=1}^{n} 1 / c_{i}} \\
y_{j}(0)=R_{j}-q_{j} \sum_{i=1}^{n}\left(R_{i}+\frac{1}{c_{i}} \log \frac{k_{i}}{k_{j}}\right)
\end{array}\right.
$$

Each company will pay a share $q_{j}$ of each claim, inversely proportional to its risk aversion. In order to compensate for the fact that the least risk-averse companies will pay greater amounts, sicle-payments or monetary compensations $y_{j}(0)$ betwcen the players occur. A consequence of the admissibility 
condition is that $\sum_{j=-1}^{n} y_{j}(0)=0$. Note that the quotas are determined by the risk aversion parameters only, so that the bargaining process will only involve the monctary compensations: another feature of exponential utilities is that the players will negotiate on amounts of money, not on abstract constants $k_{f}$.

\section{CHARACTERIZATION OF THE CORE OF THE MARIET}

Pareto-optimality has often been called group rationality: considered as a group, the members of the pool can do no better than to agree on a Paretooptimal treaty. However, this condition does not preclude the fact that some of the players might be better off by seceding and forming a sub-coalition. We are going to reduce the set of the Pareto-optimal treaties by computing the core of the game, i.c. by requiring that no sub-coalition has an incentive to quit the pool.

From now on we shall consider only Pareto-optimal treatics. Let $N$ be the set of all the companies, $S \subset N$ any sub-coalition, $v(S)$ the set of the Paretooptimal treaties for $S$, i.e. the set of all the agreements that $S$, playing separately from $N \mid S$, can achicve. $\bar{y}^{\prime}$ is said to dominate $\bar{y}$ with respect to coalition $S$ if

(i) $U_{j}\left(\bar{y}^{\prime}\right) \geqslant U_{\jmath}(\bar{y}) \quad$ for all $j \in S$ (with at least onc strict inequality)

(ii) $S$ can enforce $\bar{y}^{\prime}: \bar{y}^{\prime} \in v(S)$.

$\bar{y}^{\prime}$ is said to dominate $\bar{y}$ if there is a coalition $S$ such that $\bar{y}^{\prime}$ dominates $\bar{y}$ with respect to $S$. The core is the set of all the non-dominated treaties. In other words, instead of requiring, in addition of (1) and (2), the condition of individual rationality, we shall introduce the much stronger

\section{Condition 4: Collective rationality}

No coalition has interest in quitting the pool.

Obviously, this condition implies both conditions 2 and 3 (which are collective rationality applied, respectively, to all the one-player coalitions, and to the grand coalition $N$ ).

Assume that coalition $S \subset N$ has decided to form. Let $P_{j}^{S}$ be the exponential utility premium $C$, would require to take over a share

$$
q_{j, S}=\frac{\alpha_{j}}{\sum_{k \in S} \alpha_{k}}
$$

of the porttolio of all the companies $C_{k} \in S$, with $\alpha_{j}=\frac{1}{c_{j}}$.

In particular, $P_{j}^{\{j\}}$ (or more simply $P_{j}^{j}$ ) is the premium $c_{j}$ would demand without any reinsurance.

Let us suppose finally that all the claim amounts $\xi_{j}$ are independent. 
Lemma 1.

$$
\begin{aligned}
P_{j}^{S} & =\frac{1}{c_{j}} \sum_{k \in S} \log M_{k}\left(q_{j, S} c_{j}\right) \\
& =\frac{1}{c_{j}} \sum_{k \in S} \log M_{k}\left(\frac{1}{\sum_{j \in S} \alpha_{j}}\right) .
\end{aligned}
$$

Proof: we know that

$$
P_{j}^{S}=\frac{1}{c_{j}} \sum_{k \in S} \log M_{k}^{(*)}\left(c_{j}\right)
$$

where $M_{i}^{*(j)}(x)$ is the moment-generating function of the distribution of the quota $q_{j, S}$ of $\xi_{k}$. The fact that

$$
M_{k}^{*(f)}(x)=E\left[e^{x q_{1, s} \xi_{k}}\right]=M_{k}\left(q_{j, s} x\right)
$$

completes the proof.

\section{Lemma 2.}

Let $\left\{S_{1}, \ldots, S_{r}\right\}$ be a partition of $S \subset N$. Then

$$
\sum_{i \in S}\left(P_{j}^{S}-P_{j}^{N}\right)+\sum_{i=1}^{r} \sum_{i \in \bar{s}_{l}}\left(P_{j}^{\bar{S}_{l}}-P_{j}^{N}\right) \geqslant 0 .
$$

Proof: $\quad \sum_{i \in S}\left(P_{j}^{S}-P_{j}^{N}\right)+\sum_{i=1}^{r} \sum_{i \in \bar{s}_{l}}\left(P_{j}^{\bar{S}_{l}}-P_{j}^{N}\right)$

$$
\begin{aligned}
& =\sum_{j \in S}\left[\alpha_{j} \sum_{i \in S} \log M_{i}\left(\frac{1}{\sum \alpha_{k \in S}}\right)-\alpha_{j} \sum_{i \in N} \log M_{i}\left(\frac{1}{\sum_{k \in N} \alpha_{k}}\right)\right] \\
& +\sum_{i=1}^{r} \sum_{j \in \bar{s}_{l}}\left[\alpha_{j} \sum_{i \in \bar{s}_{l}} \log M_{i}\left(\frac{1}{\sum_{k \in \bar{s}_{l}} \alpha_{k}}\right)-\alpha_{j} \sum_{i \in N} \log M_{i}\left(\overline{\sum_{k \in N}}-\frac{1}{\alpha_{k}}\right)\right] \\
& =\sum_{i \in S}\left(\sum_{k \in S} \alpha_{k}\right) \log M_{i}\left(\frac{1}{\sum \alpha_{k} \alpha_{k}}\right)+\sum_{i=1}^{\prime} \sum_{i \in \bar{s}_{l}}\left(\sum_{k \in \bar{s}_{l}} \alpha_{k}\right) \log M_{i}\left(\frac{1}{\sum_{k \in \bar{s}_{l}} \alpha_{k}}\right) \\
& -\sum_{i \in N}\left[\sum_{k \in S} \alpha_{k}+\sum_{i=1}^{*} \sum_{i \in \bar{s}_{i}} \alpha_{j}\right] \log M_{i}\left(\frac{1}{\sum_{k \in N} \alpha_{k}}\right) \\
& =\sum_{i \in S}\left(\sum_{k \in S} \alpha_{k}\right) \log M_{i}\left(\frac{1}{\sum \alpha_{k \in S}}\right)+\sum_{i=1}^{+} \sum_{i \in \bar{s}_{l}}\left(\sum_{k \in \bar{s}_{l}} \alpha_{k}\right) \log M_{i}\left(\frac{1}{\sum_{k \in \bar{s}_{l}} \alpha_{k}}\right)
\end{aligned}
$$




$$
\begin{aligned}
& -r \sum_{i \in N}\left(\sum_{k \in \lambda} \alpha_{k}\right) \log M_{i}\left(\frac{1}{\sum_{k \in S} \alpha_{k}}\right) \quad \quad \quad\left(\text { since } \vec{S}_{l}=\bigcup_{\substack{j=1 \\
j=l}} S_{j} \cup S\right. \\
& \text { in the sequel we shall note } S_{0}=\bar{S} \text { ) } \\
& =\sum_{i \in S} a \log M_{i}\left(\frac{1}{a}\right)+\sum_{i=1}^{r} \sum_{\substack{i=0 \\
i=1}} \sum_{i \in S} a_{l} \log M_{i}\left(\frac{1}{a_{l}}\right)-r \sum_{i \in N} b \log M_{i}\left(\frac{1}{b}\right) \\
& \text { (where } a=\sum_{k \in S} \alpha_{k}, a_{l}=\sum_{k \in \bar{s}_{l}} \alpha_{k}, b=\sum_{k \in N} \alpha_{k} \text { ) } \\
& =\sum_{i \in S}\left[a \log M_{i}\left(\frac{1}{a}\right)-b \log M_{i}\left(\frac{1}{b}\right)+\sum_{i=1}^{r} \sum_{i=0}^{r} \sum_{i=s_{i}} a_{l} \log M_{i}\left(\frac{1}{a_{l}}\right)\right. \\
& -(r-1) \sum_{i \in s} b \log M_{i}\left(\frac{1}{b}\right)-r \sum_{i \in \bar{s}} b \log M_{i}\left(\frac{1}{b}\right) \\
& =\sum_{i \in S}\left[a \log M_{i}\left(\frac{1}{a}\right)-b \log M_{i}\left(\frac{1}{b}\right)\right]+\sum_{i=1}^{\infty} \sum_{\substack{i=0 \\
i=1}}^{\infty} \sum_{i \in S_{i}}\left[a_{l} \log M_{i}\left(\frac{1}{a_{l}}\right)\right. \\
& \left.-\mathrm{b} \log M_{i}\left(\frac{1}{b}\right)\right]
\end{aligned}
$$

GERBER (1974a) has shown that $\frac{1}{c} \log M(c)$ is an increasing function of $c$. It can be deduced that $c \log M\left(\frac{1}{c}\right)$ is a decreasing function of $c$. Since $a \leqslant b$ and $a_{l} \leqslant b$, all the terms between square brackets are non-negative and the lemma is proved.

\section{Corollary 1:}

For all $S \subset N$

$$
\sum_{j \in S} P_{j}^{S}+\underset{j \in S}{\Sigma} P_{j}^{\bar{S}} \geqslant \sum_{j \in N} P_{j}^{N}
$$

Proof:

Apply lemma 2 to the coalition $S=N$ and the partition $\{S, S\}$. This intuitively. obvious corollary enhances the merits of cooperation. It can be extended to all partitions of $N$. 


\section{Lemma 3:}

Let $\left\{S_{1}, \ldots, S_{r}\right\}$ be a partition of $N$. Then

$$
\sum_{i=1}^{\dot{\Sigma}} \sum_{l \in s_{l}} P_{j}^{S_{l}} \geqslant \sum_{l \in N} p_{j}^{N}
$$

which amounts to

$$
\sum_{i=1}^{r} \sum_{i \in s_{l}}\left(P_{j}^{S_{i}}-P_{j}^{N}\right) \geqslant 0
$$

Proof:

In all respects similar to lemma 2.

\section{Lemma 4:}

If $A_{1}, \ldots, A_{r}, B_{1}, \ldots, B_{r}$ are real numbers such that

$$
A_{1}+\ldots+A_{r}<B_{1}+\ldots+B_{r}
$$

there exists real numbers $\alpha_{1}, \ldots, \alpha_{r}$ such that $\alpha_{1}+\ldots+\alpha_{r}=0$ and

$$
\begin{gathered}
A_{1}+\alpha_{1}<B_{1} \\
\vdots \\
A_{r}+\alpha_{r}<B_{r} .
\end{gathered}
$$

Proof:

The property is true for $r=2$. In fact, since $A_{1}+A_{2}<B_{1}+B_{2}$, we have $A_{1}+A_{2}-B_{1}<B_{2}$, and there exists an $\varepsilon>0$ such that

$$
A_{1}+A_{2}-B_{1}+\varepsilon<B_{2} \text {. }
$$

Let $\alpha_{1}=B_{1}-A_{1}-\varepsilon$. Then

$$
\begin{aligned}
& A_{1}+\alpha_{1}=A_{1}+B_{1}-A_{1}-\varepsilon=B_{1}-\varepsilon<B_{1} \\
& A_{2}+\alpha_{2}=A_{2}-\alpha_{1}=A_{2}-B_{1}+A_{1}+\varepsilon<B_{2} .
\end{aligned}
$$

Suppose the lemma verified for a given $r$, and let us demonstrate the property for $r+1$. We have

$$
A_{1}+\ldots+A_{r+1}<B_{1}+\ldots+B_{r+1}
$$

or $A_{1}+\ldots+A_{r-1}+\left(A_{r}+A_{r+1}\right)<B_{1}+\ldots+B_{r-1}+\left(B_{r}+B_{r+1}\right)$.

There exists, by induction, $\beta_{1}, \ldots, \beta_{r}$ such that $\beta_{1}+\ldots+\beta_{r}=0$ and

$$
\begin{aligned}
& A_{1}+\beta_{1}<B_{1} \\
& \quad \vdots \\
& A_{r-1}+\beta_{r-1}<B_{r-1} \\
& \left(A_{r}+A_{r+1}\right)+\beta_{r}<B_{r}+B_{r+1} .
\end{aligned}
$$


The last incquality can be written

$$
\left(A_{r}+\beta_{r}\right)+A_{r+1}<B_{r}+B_{r+1} .
$$

There exists a $\gamma$ such that

$$
\begin{aligned}
& A_{r}+\beta_{r}+\gamma<B_{r} \\
& A_{r+1}-\gamma<B_{r+1} .
\end{aligned}
$$

It is then sufficient to put

$$
\alpha_{1}=\beta_{1}, \ldots, \alpha_{r-1}=\beta_{r-1}, \alpha_{r}=\beta+\gamma, \alpha_{r+1}=-\gamma .
$$

\section{Theorem 2:}

$\bar{y}=\left(y_{1}, \ldots, y_{n}\right)$ belongs to the core of the pool if and only if

$$
y_{j}\left(x_{1}, \ldots, x_{n}\right)=q_{j} z+y_{j}(0) \text {, with }\left\{\begin{aligned}
\sum_{i=1}^{n} y_{j}(0)=0 & \\
\sum_{i \in S} y_{j}(0) \leqslant \sum_{j \in S}\left(P_{j}^{S}-P_{j}^{N}\right), & \forall S \subset N \\
& \quad \forall \neq \phi) .
\end{aligned}\right.
$$

Proof:

(a) Necessity

Suppose $\bar{y}$ belongs to the core. It is then Pareto-optimal, and

$$
y_{j}\left(x_{1}, \ldots, x_{n}\right)=q_{j} z+y_{j}(0), \text { with } \sum_{i=1}^{n} y_{j}(0)=0 .
$$

If the last condition is not verified, therc exists a non-void $S \subset N$ such that

$$
\sum_{j \in S} y_{j}(0)>\sum_{j \in S}\left(P_{j}^{S}-P_{j}^{N}\right) \text {. }
$$

Using lemma 1 ,

$$
\sum_{j \in S} y_{j}(0)>\sum_{i \in S}\left[\frac{1}{c_{j}} \sum_{i \in S} \log M_{i}\left(\frac{1}{\sum_{k \in S} \alpha_{k}}\right)-\frac{1}{c_{j}} \sum_{i=1}^{n} \log M_{i}\left(\frac{1}{\sum_{k=1}^{k} \alpha_{k}}\right)\right]
$$

Lemma 4 makes sure that there exists $\left(z_{j}(0)_{j \in S}\right.$ such that

$$
\sum_{i \in S} z_{j}(0)=0
$$

(3) $\frac{1}{c_{j}}\left[\sum_{i \in S} \log M_{i}\left(\frac{1}{\sum_{k \in S} \alpha_{k}}\right)-\sum_{i=1}^{n} \log M_{i}\left(\frac{1}{\sum_{k=1}^{n} \alpha_{k}}\right)\right]+z_{j}(0)<y_{j}(0)$. 
Consider the sub-treaty $\bar{z}=\left(z_{j}\right)_{j \in S}$, defined by

$$
z_{j}\left[\left(x_{k}\right)_{k \in S}\right]=\left(\frac{\alpha_{j}}{\sum_{k \in S} \alpha_{k}}\right) \sum_{k \in S} x_{k}+z_{j}(0) \quad(j \in S)
$$

For all $j \in S$, we have

$$
\begin{aligned}
U_{j}(\bar{z}) & =\frac{1}{c_{j}} \int_{\theta_{S}}\left[1-e^{-c_{j}\left[R_{j}-\frac{\alpha_{j}}{\sum_{i \in S} \alpha_{k}} \sum_{k \in S} x_{k}-z_{j}(0)\right]}\right] d F_{S}(\bar{x}) \\
& =\frac{1}{c_{j}}\left[1-e^{-c_{j} R_{j}} e^{c_{j} z_{j}(0)} \int_{0_{S}} \frac{1}{e^{\frac{1}{\Sigma} \alpha_{k}} \sum_{\lambda \in S} x_{k}} d F_{S}(\bar{x})\right] \\
& =\frac{1}{c_{j}}\left[1-e^{-c_{j} R_{f}} e^{c_{j} z,(0)} \prod_{\Lambda \in S} M_{k}\left(\frac{1}{\sum_{i \in S} \alpha_{i}}\right)\right],
\end{aligned}
$$

where $0_{S}$ is the positive orthant of $E|S|$, and $I_{S}(\bar{x})$ the $|S|$ - dimensional distribution function of $\left[\left(x_{k}\right)_{k \in S}\right]$.

In the same way, we obtain

$$
U_{j}(\bar{y})=\frac{1}{c_{j}}\left[1-e^{-c_{j} R, e^{c, y_{j}(0)}} \prod_{k=1}^{n} M_{k}\left(\frac{1}{\sum_{i=1}^{n} \alpha_{i}}\right)\right] .
$$

Then $U_{j}(z)>U_{j}(\bar{y})$ if and only if

$$
e^{c, z,(0)} \prod_{\lambda \in S} M_{k}\left(\frac{1}{\sum_{i \in s} \alpha_{i}}\right)<e^{c, y,(0)} \prod_{i=1}^{n} M_{k}\left(\frac{1}{\sum_{i=1}^{n} \alpha_{i}}\right)
$$

taking logarithms

$$
\frac{1}{c_{j}}\left[\sum_{k \in S} \log M_{k}\left(\frac{1}{\sum_{i \in S} \alpha_{i}}\right)-\sum_{k=1}^{n} \log M_{k}\left(\frac{1}{\sum_{i=1}^{n} \alpha_{i}}\right)\right]+z_{j}(0)<y_{j}(0),
$$

which is precisely relation (3). So $U_{j}(z)>U_{j}(\bar{y})$, for all $j \in S$, in contradiction with the fact that $\bar{y}$ belongs to the core.

(b) Sufficiency.

Consider $y^{\prime}$ such that $y_{j}\left(x_{1}, \ldots, x_{n}\right)=q_{j} z+y_{j}(0)$, with

$$
\begin{aligned}
& \sum_{j=1}^{n} y_{j}(0)=0 \\
& \sum_{j \in S} y_{j}(0) \leqslant \sum_{j \in S}\left(P_{j}^{S}-P_{j}^{N}\right), \quad \text { for all } S \subset N(S \neq \phi)
\end{aligned}
$$


If $\bar{y}$ does not bclong to the core, there exists a coalition $S \subset N$ and a treaty $\left[\left(z_{j}\right)_{j \in S}\right]$ such that

$$
U_{j}(\bar{z}) \geqslant U_{j}(\bar{y}), \quad \forall j \in S,
$$

with a least one strict inequality. Since we can assume $\bar{z}$ to be Pareto-optimal,

$$
z_{j}\left[\left(x_{k}\right)_{k \in S}\right]=\frac{\alpha_{j}}{\sum_{k \in S} \alpha_{k}} \sum_{k \in S} x_{k}+z_{j}(0) \quad \cdot j \in S
$$

with $\sum_{j \in s} z_{j}(0)=0$.

Since $U_{j}(\bar{z})=\frac{1}{c_{j}}\left[1-c^{-c_{j} R_{j}} e^{c_{j} z_{j}(0)} \prod_{k \in S} M_{k}\left(\frac{1}{\sum_{i \in s} \alpha_{i}}\right)\right]$

and $U_{j}(\bar{y})=\frac{1}{c_{j}}\left[1-e^{-c_{j} R_{j}} e^{c_{j} y,(0)} \prod_{k=1}^{n} M_{k}\left(\frac{1}{\sum_{i=1}^{n} \alpha_{i}}\right)\right] \quad \forall j \in S$.

We have, taking logarithms,

$z_{j}(0)+\frac{1}{c_{j}} \sum_{i \in S} \log M_{k}\left(\frac{1}{\sum_{i \in, s} \alpha_{i}}\right) \leqslant y_{j}(0)+\frac{1}{c_{j}} \sum_{i=1}^{n} \log M_{k}\left(\sum_{\sum_{i=1}^{n} \alpha_{i}}^{1}\right) \forall j \in S$.

Summing over all $j \in S$, and using $\sum_{j \in S} z_{j}(0)=0$, we obtain

$\sum_{, \in S} y_{j}(0)>\sum_{j \in s}\left[\frac{1}{c_{j}} \sum_{k \in S} \log M_{k}\left(\frac{1}{\sum_{i \in S} \alpha_{i}}\right)-\frac{1}{c_{j}} \sum_{k=1}^{n} \log M_{k}\left(\frac{1}{\sum_{i=1}^{n} \alpha_{i}}\right)\right]$

or

$$
\sum_{j \in S} y_{j}(0)>\sum_{j \in S}\left[P_{j}^{S}-P_{j}^{N}\right]
$$

contradicting the hypothesis.

Corollary.

$\bar{y}=\left(\bar{y}_{1}, \ldots, y_{n}\right)$ belongs to the core of the pool if and only if

$$
y_{j}\left(x_{1}, \ldots, x_{n}\right)=q_{j} z+y_{j}(0), \quad j=1, \ldots, n
$$

with $\sum_{j \in S} y_{j}(0) \leqslant \sum_{j \in S}\left(P_{j}^{S}-P_{j}^{N}\right) \quad \forall S \subset N$

if we define $P_{j}^{\infty}=0$. 
Proof:

Applying condition $\sum_{j \in S} y_{j}(0) \leqslant \sum_{j \in S}\left(P_{j}^{S}-P_{j}^{N}\right)$ for $S=N$, we obtain

$$
\sum_{j \in N} y_{j}(0)=\sum_{j=1}^{n} y_{j}(0) \leqslant \sum_{j \in N}\left(P_{j}^{N}-P_{j}^{N}\right)=0
$$

Since $\sum_{j \in \mathscr{Q}}\left(P_{j}^{\mathrm{o}}-P_{j}^{N}\right)=0$ and $\sum_{j \in \mathcal{O}} y_{j}(0)+\sum_{j \in N} y_{j}(0)=0$, we have successively

$$
\begin{aligned}
& \sum_{j=1}^{n} y_{j}(0)=0 \\
& \sum_{j \in N} y_{j}(0) \geqslant 0 \\
& \sum_{j \in \infty} y_{j}(0) \leqslant 0=\sum_{j \in \infty}\left(P_{j}^{\infty}-P_{j}^{N}\right)
\end{aligned}
$$

In other words, condition $\sum_{i=1}^{n} y_{j}(0)=0$ may be replaced by

$$
\begin{aligned}
& \sum_{j \in N} y_{j}(0) \leqslant \sum_{j \in N}\left(P_{j}^{N}-P_{j}^{N}\right) \\
& \sum_{i \in \varnothing} y_{j}(0) \leqslant \sum_{j \in \varnothing}\left(P_{j}^{\sigma}-P_{j}^{N}\right) .
\end{aligned}
$$

So, not only conditions 2 and 3 , but also condition 1 derives from collective rationality.

\section{Interpretation:}

In addition to the fact that it characterizes the core, theorem 2 may be interesting in the sense that it links two apparently very different concepts, a collective notion (the core of a game without transferable utilities), and an individual notion (a premium calculation principle).

Applied to a one-player coalition, the core condition becomes

$$
y_{j}(0) \leqslant P_{j}^{j}-P_{j}^{N} \quad j=1, \ldots, n .
$$

The second member is the difference between the exponential utility premiums before and after reinsurance. Everything happens as if each company evaluates its portfolio by the exponential utility premium: the certainty equivalent of any portfolio is this premium. A positive second member means that $C_{f}$ finds profitable to participate to the pool. It will however only enter the market if its "fee" or side-payment does not exceed the profit.

Applied to the two-player coalition $\{1,2\}$, the core condition becomes

$$
y_{1}(0)+y_{2}(0) \leqslant\left[P_{1}^{\{1,2\}}-P_{1}^{N}\right]+\left[P_{2}^{\{1,2\}}-P_{2}^{N}\right]
$$


$P_{1}^{\{1,2\}}$ is the premium $C_{1}$ would ask to assume a share $\frac{\alpha_{1}}{\alpha_{1}+\alpha_{2}}$ of its own and $C_{2}$ 's portfolios. The term between the first square brackets represents the positive or negative profit $C_{1}$ would make by not seceding from the pool with $C_{2}$. The condition requires that, globally, coalition $\{1,2\}$ has no interest to play alone, in the sense that the sum of the side payments required from its members is small enough not to incite them to quit the pool. The difference

$$
\left[P_{1}^{\{1,2\}}-P_{1}^{N}\right]+\left[P_{2}^{\{1,2\}}-P_{1}^{N}\right]-y_{1}(0)-y_{2}(0)
$$

is the bencfit coalition $\{1,2\}$ enjoys from participating to the pool. If this term were negative, $\{1,2\}$ would have interest to scparate and create a 2 company pool. Note that nothing is said about the way those 2 compranics will share this benefit: the core only introduces global conditions.

Note:

The conditions of theorem 2 not only provide upper limits for the side-payments, but also lower limits. Indeed, since

$$
\sum_{j \in S} y_{j}(0)=-\sum_{j \in \bar{s}} y_{j}(0) \quad \forall S,
$$

we lave

$$
-\sum_{j \in \bar{S}}\left(P_{j}^{\bar{S}}-P_{j}^{N}\right) \leqslant \sum_{j \in S} y_{j}(0) \leqslant \sum_{\epsilon \in S}\left(P_{j}^{S}-P_{j}^{N}\right)
$$

3. EXISTENCE OF THE CORE

The main disadvantage of the core is that there exists large classes of games for which it is empty. Fortunately, theorem 3 shows that the core of the risk exchange market always exists.

\section{Theorem 3:}

The core of the market is non-void.

Proof:

The core can be characterized by conditions (3), or, in an equivalent way, by

$$
\begin{cases}\sum_{j \in \bar{S}}\left(P_{j}^{\bar{S}}-P_{j}^{N}\right) \leqslant \sum_{j \in S} y_{j}(0) \leqslant \sum_{j \in S}\left(P_{j}^{S}-P_{j}^{N}\right) & \text { for all } S \subset N \\ y_{1}(0)+\ldots+y_{n}(0)=0 & \text { such that } C_{n} \notin S\end{cases}
$$

Note that conditions (4) only restrict the values of $y_{1}(0), \ldots, y_{n-1}(0)$. This is obvious because, if $C_{n} \in S_{0}$,

$$
\sum_{j \in S_{0}} y_{j}(0)=-\sum_{i \in \bar{s}_{0}} y_{j}(0) \text { and } C_{n} \notin \bar{S}_{0},
$$


So

$$
-\sum_{j \in s_{0}}\left(P_{j}^{S_{0}}-P_{j}^{N}\right) \leqslant \sum_{j \in \bar{S}_{0}} y_{j}(0) \leqslant \sum_{j \in \bar{s}_{0}}\left(P_{j}^{\bar{S}_{0}}-P_{j}^{N}\right)
$$

and

$$
-\sum_{j \in \bar{s}_{0}}\left(P_{j}^{\bar{S}_{0}}-P_{j}^{N}\right) \leqslant \sum_{i \in s_{0}} y_{j}(0) \leqslant \sum_{j \in s_{0}}\left(P_{j}^{S_{0}}-P_{j}^{N}\right) \text {. }
$$

It only remains to prove that there exists $(n-1)$ constants $y_{1}(0), \ldots$, $y_{n-1}(0)$ verifying conditions (4) (then we shall obtain $y_{n}(0)$ using $y_{n}(0)=$ $\left.-\sum_{j=1}^{n-1} y_{j}(0)\right)$. This system has a solution if, for all $S \subset N$ such that $C_{n} \notin S$ and for all partitions $\left(S_{1}, \ldots, S_{r}\right)$ of $S$,

$$
\begin{aligned}
- & \sum_{, \in \bar{S}}\left(P_{j}^{\bar{S}}-P_{j}^{N}\right) \leqslant \sum_{j \in S}\left(P_{j}^{S}-P_{j}^{N}\right) \\
& \sum_{j \in S}\left(P_{j}^{S}-P_{j}^{N}\right) \geqslant-\sum_{i=1} \sum_{i \in \bar{s}_{l}}\left(P_{j}^{\bar{S}_{l}}-P_{j}^{N}\right) \\
- & \sum_{j \in \bar{S}}\left(P_{j}^{\bar{S}}-P_{j}^{N}\right) \leqslant \sum_{i=1} \sum_{j \in s_{l}}\left(P_{j}^{S_{l}}-P_{j}^{N}\right) .
\end{aligned}
$$

This is a consequence of corollary 1 and lemmas 2 and 3 .

\section{EXAMPLE}

Let us consider the following example, introduced by LemAIRE (1979). Suppose that the pool consists of 3 companies, whose risk aversion coefficients are respectively $c_{1}=.3, c_{2}=.6, c_{3}=.1$. All the companies have the same distribution of claim amounts, namely a $\Gamma$-distribution

$$
\frac{d F_{j}(x)}{d x}=\left\{\begin{array}{ll}
\frac{\tau^{a}}{\Gamma(a)} e^{-\tau x} x^{a-1} & x>0 \\
0 & \text { elsewhere }
\end{array} j=1,2,3,\right.
$$

with parameters $a=1.15^{2}$ and $\tau=.96$. The mean is equal to $m=\frac{a}{\tau}=1.2$, the variance $\sigma^{2}=\frac{a}{\tau^{2}}=1.25$. Using the moment-gcnerating function of this distribution

$$
M(t)=\left(1-\frac{t}{\tau}\right)^{-a}
$$

we obtain the following conditions for the core

$$
\begin{aligned}
& .388 \leqslant y_{1}(0) \leqslant . .610 \\
& .818 \leqslant y_{2}(0) \leqslant 1.469 \\
& 1.22 \leqslant y_{1}(0)+y_{2}(0) \leqslant 1.448 .
\end{aligned}
$$


The core is shown in Figure 1. Note that the core (hachured area) is substantially smallcr than the set of the Pareto-optimal treaties (dotted area).

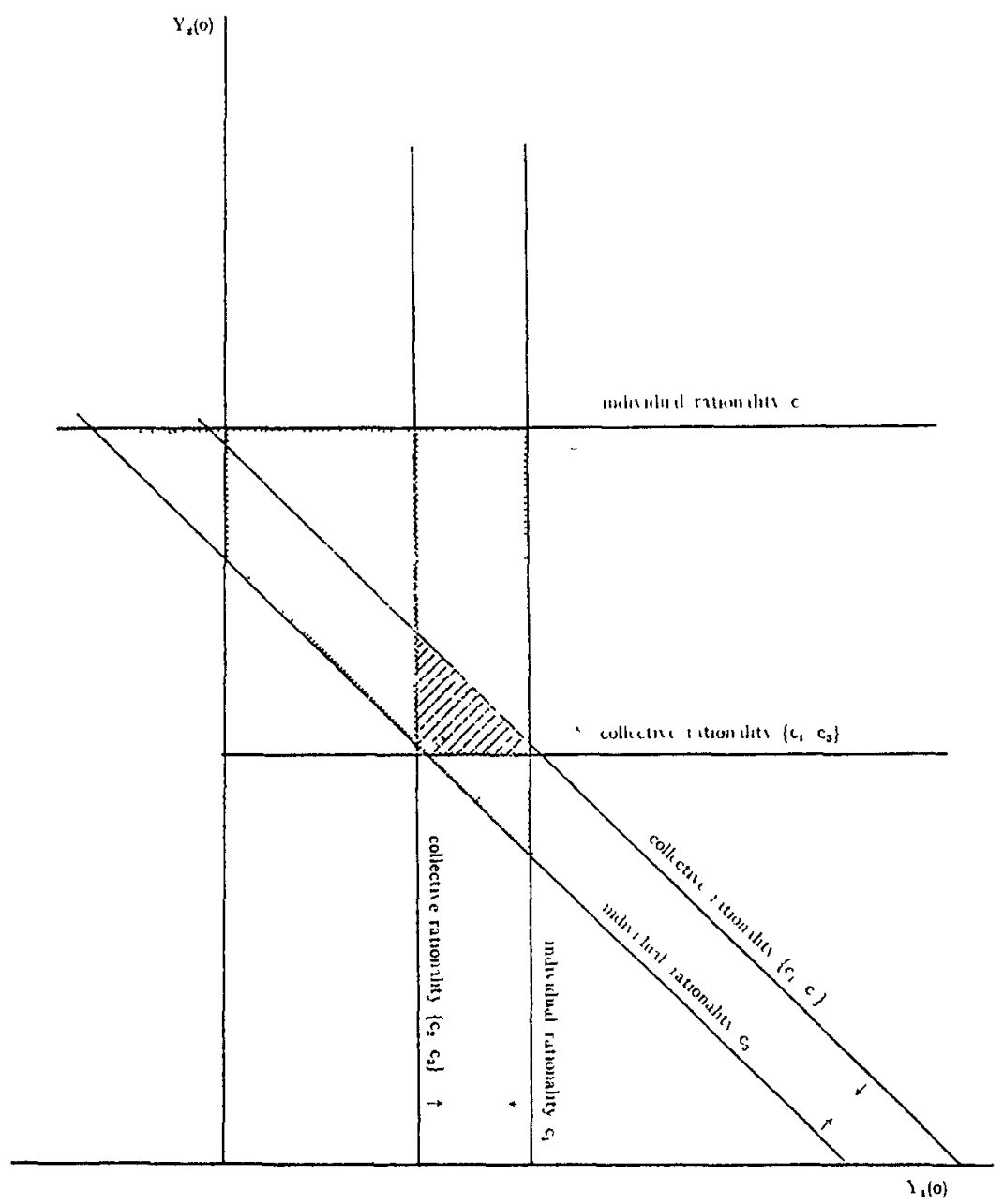

Note:

For $n>3$, the core is more difficult to represent, since it forms a convex compact polyhedron in the $n$-1-dimensional Euclidian space with axis $y_{1}(0)$, $\ldots, y_{n-1}(0)$ It is characterized by a set of $2^{n-1}-1$ double inequalities. This number of constraints increases tremendously with $n$. 
REFERENCES

Borch, K. (1900a). Reciprocal reinsurance treaties seen as a two-person cooperative game. Skandinavish Ahtuarietidshrift 43, 29-58.

Borc1, K. (1960)). Reciprocal reinsurance treaties. Astin Bulletin 1, $170-191$.

Borcu, K. (1961). The utility concept applied to the theory of insurance. A stin Bulletin 1, $245-255$.

Bokci, K. (1962). Equilibrium in a reinsurance market. Econometrica 30, 424-444.

Bühlman, H. (1970). Mathematical methods in risk theory. Springer-Verlag, Berlin.

Du Mouchel, W. (1968). The Pareto-optimality of an 11 -company reinsurance trcaty. Shandinavisk Aktuarietidshrift 51, 165-170.

GERBER, H. (1974a). On additive premium calculation principles. Astin Bulletin 7, 21 5-222.

Grerber, H. (1974b). On iterative premium calculation principles. Mitteilungen der Vercinigung Schweizerischer Versicherungsmathematiker 74, 163-172.

Lieple, P. (1975). Uber die Wahl von Nutzenfunktionen für die Bestimmung von Versichcrungsprämien. Mitteilungen der Vereinigung Schweizerischer Versicherungsmathematiker 75, 27-45.

LEMAirE, J. (1973). Optimalité d'un contrat d'échange de risques entre assureurs. Cahiers du C.E.R.O. 15, 139.156.

LEMAIRE, J. (1979). A non-symmetrical value for games without transferable utilities, application to reinsurance. Astin Bulletin 10, 195-214. 